\title{
Long-term prognosis in patients with acute myocardial infarction and newly detected glucose abnormalities: predictive value of oral glucose tolerance test and $\mathrm{HbA} 1 \mathrm{c}$
}

Stelios Karayiannides ${ }^{1,2^{*}+}$ (D), Catarina Djupsjö ${ }^{3,4+}$, Jeanette Kuhl ${ }^{3,5}$, Claes Hofman-Bang ${ }^{6}$, Anna Norhammar ${ }^{3,7}$, Martin J. Holzmann ${ }^{3,8}$ and Pia Lundman ${ }^{1,6}$

\begin{abstract}
Background: Disturbances of glucose metabolism can be diagnosed by an oral glucose tolerance test (OGTT) and by glycated haemoglobin ( $\mathrm{HbA1c}$ ). The aim of this study was to investigate the association between newly detected disturbances of glucose metabolism and long-term prognosis after acute myocardial infarction (AMI) and to compare the predictive value of an OGTT and HbA1c.

Methods: Patients under the age of 80 years with no known history of diabetes admitted for AMI at the Department of Cardiology, Danderyd University Hospital, Stockholm, Sweden, from January 1st, 2006 until December 31st, 2013, were investigated with an OGTT and a HbA1c before discharge and were classified as having normal glucose tolerance (NGT), prediabetes or diabetes according to American Diabetes Association (ADA) criteria. Using nationwide, all-inclusive registers, patients were followed for the incidence of combined event [CE (first of myocardial infarction, heart failure, ischaemic stroke or mortality)] for a mean follow-up time of 4.8 years. Cox regression analysis was used to calculate Hazard Ratios (HR) and their 95\% confidence intervals (Cl).

Results: Of the 841 patients who were investigated with both an OGTT and a HbA1c, 139 (17\%) patients had NGT, 398 (47\%) had prediabetes and 304 (36\%) had diabetes according to OGTT. The corresponding figures using HbA1c were 320 (38\%), 461 (55\%) and 60 (7\%). Patients with newly discovered diabetes were older and had a higher body mass index compared to those with NGT. OGTT was not predictive for CE. In contrast, prediabetes identified by a $\mathrm{HbA1} \mathrm{c}$ was associated with an increased risk for CE (HR 1.31; 95\% Cl 1.05-1.63) compared to normoglycaemia. When comparing the prognostic value of different glucose and $\mathrm{HbA1c}$ cut-offs, only a $\mathrm{HbA} 1 \mathrm{c} \geq 39 \mathrm{mmol} / \mathrm{mol}$ was significantly associated with CE (HR 95\% Cl; 1.30:1.05-1.61).

Conclusion: In this single-centre study, in a recent contemporary cohort, we found that around two thirds of the patients admitted with AMI with no known history of diabetes had disturbed glucose metabolism, in accordance with previous studies. $\mathrm{HbA1c}$ in the prediabetes range, but not OGTT, added predictive value on the long-term outcome, in a cohort to whom a pathologic OGTT result was communicated with lifestyle advice.
\end{abstract}

\footnotetext{
*Correspondence: stelios.karayiannides@ki.se

${ }^{\dagger}$ Stelios Karayiannides and Catarina Djupsjö contributed equally to this work

1 Department of Clinical Sciences, Karolinska Institutet, Danderyd Hospital, SE-182 88 Stockholm, Sweden

Full list of author information is available at the end of the article
}

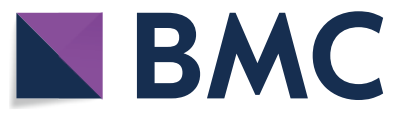

(c) The Author(s) 2021. This article is licensed under a Creative Commons Attribution 4.0 International License, which permits use, sharing, adaptation, distribution and reproduction in any medium or format, as long as you give appropriate credit to the original author(s) and the source, provide a link to the Creative Commons licence, and indicate if changes were made. The images or other third party material in this article are included in the article's Creative Commons licence, unless indicated otherwise in a credit line to the material. If material is not included in the article's Creative Commons licence and your intended use is not permitted by statutory regulation or exceeds the permitted use, you will need to obtain permission directly from the copyright holder. To view a copy of this licence, visit http://creativeco mmons.org/licenses/by/4.0/. The Creative Commons Public Domain Dedication waiver (http://creativecommons.org/publicdomain/ zero/1.0/) applies to the data made available in this article, unless otherwise stated in a credit line to the data. 
Keywords: Myocardial infarction, Diabetes, Prediabetes, Oral glucose tolerance test, Hemoglobin A1c, Prognosis

\section{Background}

Diabetes mellitus is an established risk factor for cardiovascular disease and mortality and patients with diabetes have a worse outcome following acute coronary events [1-3]. After acute myocardial infarction (AMI), patients with both known diabetes as well as newly detected abnormal glucose metabolism, have been shown to have a poorer long-term outcome with increased risk for mortality and future cardiovascular events than patients with normal glucose metabolism [4-6]. This may reflect the fact that patients with diabetes are undiagnosed for many years and present with a cardiovascular complication, but could also indicate that the increased cardiovascular risk associated with elevated glucose levels begins before the diagnosis of diabetes [7].

Disturbances of glucose metabolism can be detected by different methods. The oral glucose tolerance test (OGTT) has been the golden standard for many years [8] and haemoglobin A1c (HbA1c) was approved as diagnostic tool in 2009 [9]. Diagnostic criteria for glucose disturbances differ slightly between American Diabetes Association (ADA) and World Health Organisation (WHO) with regards to the cut-off for impaired fasting glycaemia (IFG) and the prediabetes cut-off for HbA1c [10]. To detect prediabetes and diabetes, WHO recommends performing an OGTT, while ADA recommends using a $\mathrm{HbAlc}$, partly because it is less time consuming and therefore more cost-effective. However, there are indications that a HbAlc and an OGTT identify different populations with different risks, [11, 12 ] and it is yet not clarified if one method is superior to the other in predicting long-term prognosis. Shahim et al., showed that a 2-h postload OGTT glucose (2 h-PG) was a better prognostic test regarding cardiovascular events in patients with established coronary artery disease, but with a mean follow-up time of only 2 years [13]. Another study found that 2 h-PG was an indicator of a poorer prognosis in people in general, but in this study no comparison to HbA1c or outcome in cardiovascular patients was specifically made [14]. The latest European Society of Cardiology guidelines on diabetes, pre-diabetes and cardiovascular diseases state that there is a gap in evidence with regards to whether HbA1c or an OGTT is the best prognostic marker regarding important outcomes in patients with coronary heart disease [15].

The aim of the present study was to investigate the association between newly detected disturbances of glucose metabolism and long-term prognosis after AMI and to compare the predictive value of an OGTT and a HbA1c.

\section{Methods \\ Study design}

This was a single-centre, observational, cohort study. An individual consent for inclusion in the study was not collected. The Swedish Patient Data Act allows the inclusion of personal data in a registry for the purpose of improving the quality of care, as long the individual is informed and has the right of opting out at any time and having his/her personal data erased.

The study complied with the Declaration of Helsinki and was approved by the regional research ethics committee in Stockholm, Sweden (reg. no 2014/338-31/2 and 2018/1321-32).

\section{Study population and data sources}

Following publications from Sweden indicating a high proportion of glucose disturbances in patients with AMI [16], the Department of Cardiology at Danderyd University Hospital in Stockholm, Sweden, initiated an implementation program to identify undetected dysglycaemia and offered lifestyle advice and follow-up of these patients. From January 1st, 2006 until December 31st, 2013, 3417 patients with acute coronary syndrome under the age of 80 years were admitted to the Department of Cardiology at Danderyd University Hospital. As part of the usual clinical care, the majority of patients with no known history of diabetes were screened for undiagnosed glucose disturbances with a standardised 75-g OGTT after an overnight fast, with plasma glucose values analysed at 0 and $120 \mathrm{~min}$, no earlier than four days after admission. HbA1c was not routinely taken simultaneously in all patients, particularly at the beginning of the study period. Using information from the Swedish Web-system for Enhancement and Development of Evidence-based care in Heart disease Evaluated According to Recommended Therapies (SWEDEHEART), [17] a national quality registry that contains data on all patients with acute coronary syndromes, we first identified all the patients that were admitted within our inclusion period. Patient records were then examined with regards to OGTT and HbA1c values. We excluded 433 AMI patients with a known history of diabetes and 1300 patients who were not screened for diabetes, most of them due to clinical reasons. In total, 1684 patients with AMI were screened with an OGTT and 841 (50\%) 
of them also had a registered HbA1c value, which was analysed concurrently with the OGTT (Additional file 1: Figure S1). These 841 patients made up our study cohort.

By using the unique personal identity number [18] that every Swedish resident has, data regarding comorbidities, medications and all-cause mortality were collected from four different sources: (1) Swedish Web-system for Enhancement and Development of Evidence-based care in Heart Disease Evaluated According to Recommended Therapies (SWEDEHEART) register, which contains information on patients admitted at Swedish hospitals with acute coronary syndrome and of all the patients undergoing coronary angiography, percutaneous coronary intervention or coronary artery bypass grafting, (2) the National Patient Register (NPR), which includes information on diagnoses according to the ICD-10 system for both inpatient care and outpatient specialist visits, (3) the Prescribed Drug Register, which contains data on all filled prescriptions since July 1st 2005 and (4) the Cause of Death Register, which includes information on date and cause of death for all Swedish residents.

All patients were followed up for all-cause mortality until December 25th, 2017 and for hospitalisation for myocardial infarction, ischaemic stroke or heart failure until December 31st, 2014. We report on the incidence of combined event [CE (first of myocardial infarction, heart failure, ischaemic stroke or mortality)]. The mean follow-up time for our study population was 4.8 years. Information on dispensed prescription medications were collected from 5 months before hospitalisation with AMI to 1 month after discharge.

\section{Definitions}

Based on the results of the OGTT, patients were categorised into three groups according to ADA criteria: [19] (1) Normoglycaemia (NGT), (2) Prediabetes [Impaired glucose tolerance (IGT), Impaired fasting glucose (IFG) or both] and (3) Type 2 diabetes. HbA1c was also used to categorise the patients into three groups according to ADA criteria: (1) Normoglycaemia (NGT), (2) Prediabetes, and (3) Type 2 diabetes (Additional file 1: Table S1). Previously known diabetes was defined as self-reported history in SWEDEHEART. A complete list of all the definitions used in the study can be found in the online supplement (Additional file 1: Table S2).

\section{Statistical methods}

Baseline characteristics are presented as medians with interquartile range for continuous and absolute numbers and percentage for categorical variables. Baseline characteristics stratified by glucose perturbation group were compared using the chi-square or the non-parametric Kruskal-Wallis test as applicable. Time-to-event rates were calculated using survival analysis. Cox regression analysis was used to calculate Hazard Ratios (HR) and their 95\% confidence intervals (CI) for all-cause mortality. The proportionality of hazards assumption was not violated when tested using Schoenfeld residuals. A twosided significance level of $\mathrm{p}<0.05$ was considered significant for all tests. Statistical analysis was performed using STATA version 14 (STATA Corp., College Station, TX, USA).

\section{Results \\ Baseline characteristics}

Baseline characteristics of the 841 patients making up the study population, with OGTT and HbA1c results analysed are shown in Tables 1 and 2.

\section{Glucose disturbances by OGTT result}

When investigated with an OGTT, a total of 139 (17\%) patients had NGT, 398 (47\%) had prediabetes (IGT and/ or IFG) and 304 (36\%) had newly discovered diabetes. Patients with newly discovered diabetes were older and had a higher BMI than patients with NGT and prediabetes. Patients with newly discovered diabetes were significantly less often treated with ticagrelor compared to patients with NGT and prediabetes. There were no significant differences in comorbidities or treatment with aspirin, clopidogrel, ACE-inhibitors, beta-receptor blockers or statins between the three groups.

Baseline characteristics of all patients who underwent an OGTT $(\mathrm{n}=1684)$ are shown in Additional file 1 : Table S3.

\section{Glucose disturbances by $\mathrm{HbA} 1 \mathrm{c}$ results}

When patients were categorised into groups according to HbA1c results, 320 (38\%) patients had a normal HbA1c (<39 $\mathrm{mmol} / \mathrm{mol}), 461$ (55\%) had prediabetes (HbA1c $39-47 \mathrm{mmol} / \mathrm{mol})$ and $60(7 \%)$ were defined as having diabetes $(\mathrm{HbA} 1 \mathrm{c} \geq 48 \mathrm{mmol} / \mathrm{mol})$. Similar findings were observed as with the OGTT, where patients with newly discovered diabetes were older and had a higher BMI than the other two groups. Those with diabetes were significantly less often treated with angiotensin II receptor-blockers and ticagrelor compared with patients with NGT and prediabetes. We observed no other significant differences in comorbidities or medications between the three groups.

The numbers (and percentages) of patients in the different glucose groups according OGTT and HbA1C results are illustrated in Fig. 1.

\section{Comparison between OGTT and $\mathrm{HbA1c}$ results}

Only 87 (10\%) out of 841 patients had fasting plasma glucose (fPG), $2 \mathrm{~h}-\mathrm{PG}$ and HbA1c values within the 
Table 1 Baseline characteristics of our study population stratified by glycaemic status according to OGTT results (ADA criteria)

\begin{tabular}{|c|c|c|c|c|c|c|}
\hline Characteristic & Available data & NGT & Prediabetes (IFG/IGT) & Diabetes & p-value & Known diabetes \\
\hline Study population —no known diabetes, $n=841$ & & $139(16.5 \%)$ & $398(47.3 \%)$ & $304(36.2 \%)$ & & 433 \\
\hline Age-yr [median (IQR)] & $841 / 841$ & $63(54-69)$ & $64(58-71)$ & $66(58-71)$ & 0.019 & $68(61-75)$ \\
\hline Gender (male) & $841 / 841$ & $104(74.8 \%)$ & $304(76.4 \%)$ & $212(69.7 \%)$ & 0.133 & $314(72.5 \%)$ \\
\hline Body-mass index [median (IQR)] & $796 / 841$ & $25(23-28)$ & $27(24-30)$ & $28(25-30)$ & $<0.001$ & $28(26-32)$ \\
\hline Smokers & $801 / 841$ & $38(29.5 \%)$ & $142(37.3 \%)$ & $118(40.5 \%)$ & 0.054 & $84(21.9 \%)$ \\
\hline Snuff users & $560 / 841$ & $2(2.0 \%)$ & $23(8.4 \%)$ & $12(6.5 \%)$ & 0.083 & $9(3.5 \%)$ \\
\hline $\begin{array}{l}\text { eGFR (mL/min/1.73 m2)—MDRD equation [median } \\
\text { (IQR)] }\end{array}$ & $769 / 841$ & $81(69-89)$ & $83(72-97)$ & $83(70-96)$ & 0.177 & $73(55-92)$ \\
\hline \multicolumn{7}{|l|}{ Medical history } \\
\hline Previous myocardial infarction & $841 / 841$ & $40(28.8 \%)$ & $101(25.4 \%)$ & $63(20.7 \%)$ & 0.143 & $103(23.8 \%)$ \\
\hline Hypertension & $841 / 841$ & $36(25.9 \%)$ & $88(22.1 \%)$ & $71(23.4 \%)$ & 0.658 & $179(41.3 \%)$ \\
\hline Heart failure & $841 / 841$ & $3(2.2 \%)$ & $5(1.3 \%)$ & $3(1.0 \%)$ & 0.598 & $14(3.2 \%)$ \\
\hline Atrial fibrillation & $841 / 841$ & $8(5.8 \%)$ & $14(3.5 \%)$ & $13(4.3 \%)$ & 0.520 & $26(6.0 \%)$ \\
\hline Previous $\mathrm{PCl}$ or $\mathrm{CABG}$ & $841 / 841$ & $32(23.0 \%)$ & $84(21.1 \%)$ & $58(19.1 \%)$ & 0.612 & $83(19.2 \%)$ \\
\hline Stroke & $841 / 841$ & $5(3.6 \%)$ & $8(2.0 \%)$ & $12(3.9 \%)$ & 0.291 & $23(5.3 \%)$ \\
\hline Peripheral artery disease & $841 / 841$ & $2(1.4 \%)$ & $6(1.5 \%)$ & $3(1.0 \%)$ & 0.825 & $13(3.0 \%)$ \\
\hline Chronic kidney disease & $841 / 841$ & $2(1.4 \%)$ & $2(0.5 \%)$ & $2(0.7 \%)$ & 0.523 & $14(3.2 \%)$ \\
\hline Chronic obstructive pulmonary disease & $841 / 841$ & $3(2.2 \%)$ & $3(0.8 \%)$ & $3(1.0 \%)$ & 0.377 & $8(1.8 \%)$ \\
\hline \multicolumn{7}{|l|}{ Medications on discharge } \\
\hline Aspirin & $841 / 841$ & $121(87.1 \%)$ & $342(85.9 \%)$ & $246(80.9 \%)$ & 0.121 & $280(64.7 \%)$ \\
\hline Clopidogrel & $841 / 841$ & $79(56.8 \%)$ & $225(56.5 \%)$ & $188(61.8 \%)$ & 0.334 & $190(43.9 \%)$ \\
\hline Ticagrelor & $841 / 841$ & $44(31.7 \%)$ & $95(23.9 \%)$ & $46(15.1 \%)$ & $<0.001$ & $56(12.9 \%)$ \\
\hline Prasugrel & $841 / 841$ & $0(0 \%)$ & $4(1.0 \%)$ & $3(1.0 \%)$ & 0.497 & $8(1.9 \%)$ \\
\hline ACE-inhibitors & $841 / 841$ & $89(64.0 \%)$ & $257(64.6 \%)$ & $189(62.2 \%)$ & 0.802 & $202(46.7 \%)$ \\
\hline$A R B$ & $841 / 841$ & $25(18.0 \%)$ & $65(16.3 \%)$ & $54(17.8 \%)$ & 0.845 & $95(21.9 \%)$ \\
\hline Beta-receptor blockers & $841 / 841$ & $126(90.7 \%)$ & $341(85.7 \%)$ & $251(82.6 \%)$ & 0.08 & $277(64.0 \%)$ \\
\hline Statins & $841 / 841$ & $122(87.8 \%)$ & 342 (85.9\%) & 252 (82.9\%) & 0.338 & $271(62.6 \%)$ \\
\hline Dihydropyridines & $841 / 841$ & $22(15.8 \%)$ & $70(17.6 \%)$ & $69(22.7 \%)$ & 0.129 & $111(25.6 \%)$ \\
\hline Diuretics & $841 / 841$ & $15(10.8 \%)$ & $65(16.3 \%)$ & $59(19.4 \%)$ & 0.076 & 101 (23.3\%) \\
\hline Warfarin & $841 / 841$ & $7(5.0 \%)$ & $21(5.3 \%)$ & $14(4.6 \%)$ & 0.921 & $29(6.7 \%)$ \\
\hline
\end{tabular}

normoglycaemic range. Of the 754 patients with dysglycaemia (prediabetes or diabetes), $425(56 \%)$ were identified by a single fPG, 636 (84\%) by a 2 h-PG and $521(69 \%)$ by a HbA1c. The OGTT (fPG and 2 h-PG) identified 702 patients $(93 \%)$ as having dysglycaemia and the combination of fPG and HbA1c identified 626 patients $(83 \%)$. Screening for dysglycaemia with OGTT and HbA1c identified different at-risk groups. Only 281 (37\%) patients had values showing dysglycaemia as identified by both fPG, 2 h-PG and HbA1c. The concordance of the OGTT and a HbA1c in classifying dysglycaemia was modest, with only $62 \%$ of patients classified as having dysglycaemia on both the OGTT and HbA1c (Fig. 2).

\section{Event rates and long-term outcome in patient groups according to OGTT results}

Of the study population of 841 patients, 108 (12.8\%) died during a mean follow-up time of 6.9 years. With regards to cardiovascular events, $175(20.8 \%)$ patients suffered a myocardial infarction, $19(2.3 \%)$ patients had an ischaemic stroke, and 120 (14.3\%) patients were hospitalised for heart failure. The total number of combined events (first of myocardial infarction, stroke, heart failure or mortality) was 372 (44.2\%).

Regarding the incidence of CE, 58 (41.7\%) patients with NGT had events, 174 (43.7\%) events occurred in the prediabetes group and 140 (46.1\%) events in the group with patients with newly discovered diabetes. There was no significant difference in hazard ratios neither for the CE nor for all-cause mortality between the three patient groups, both unadjusted and adjusted for age and sex (Table 3 and Additional file 1: Table S4). Figure 3A shows a Kaplan-Meier curve with freedom from $C E$ and Additional file 1: Figure S2A shows a Kaplan-Meier curve with freedom from all-cause mortality between the three groups, categorised according to OGTT results. 
Table 2 Baseline characteristics of our study population stratified by glycaemic status according to HbA1c results (ADA criteria)

\begin{tabular}{|c|c|c|c|c|c|c|}
\hline Characteristic & Available data & NGT & Prediabetes & Diabetes & p-value & Known diabetes \\
\hline Study population—no known diabetes, $n=841$ & & $320(38.1 \%)$ & $461(54.8 \%)$ & $60(7.1 \%)$ & & 433 \\
\hline Age-yr [median (IQR)] & $841 / 841$ & $63(56-70)$ & $66(59-71)$ & $66(57-72)$ & 0.026 & $68(61-75)$ \\
\hline Gender (male) & $841 / 841$ & $242(75.6 \%)$ & $334(72.5 \%)$ & $44(73.3 \%)$ & 0.610 & $314(72.5 \%)$ \\
\hline Body-mass index [median (IQR)] & $796 / 841$ & $26(24-29)$ & $27(25-30)$ & $29(25-32)$ & $<0.001$ & $28(26-32)$ \\
\hline Smokers & $801 / 841$ & $103(33.1 \%)$ & $169(39.1 \%)$ & $26(44.8 \%)$ & 0.035 & $84(21.9 \%)$ \\
\hline Snuff users & $560 / 841$ & $11(4.9 \%)$ & $26(8.9 \%)$ & $0(0 \%)$ & 0.034 & $9(3.5 \%)$ \\
\hline eGFR (mL/min/1.73 m2)—MDRD equation [median (IQR)] & $769 / 841$ & $85(71-96)$ & $81(71-94)$ & $85(76-98)$ & 0.104 & $73(55-92)$ \\
\hline \multicolumn{7}{|l|}{ Medical history } \\
\hline Previous myocardial infarction & $841 / 841$ & $82(25.6 \%)$ & $108(23.4 \%)$ & $14(23.3 \%)$ & 0.769 & $103(23.8 \%)$ \\
\hline Hypertension & $841 / 841$ & $72(22.5 \%)$ & $110(23.9 \%)$ & $13(21.7 \%)$ & 0.869 & $179(41.3 \%)$ \\
\hline Heart failure & $841 / 841$ & $3(0.9 \%)$ & $7(1.5 \%)$ & $1(1.7 \%)$ & 0.756 & $14(3.2 \%)$ \\
\hline Atrial fibrillation & $841 / 841$ & $10(3.1 \%)$ & $21(4.6 \%)$ & $4(6.7 \%)$ & 0.371 & $26(6.0 \%)$ \\
\hline Previous $\mathrm{PCl}$ or $\mathrm{CABG}$ & $841 / 841$ & $64(20.0 \%)$ & $99(21.5 \%)$ & $11(18.3 \%)$ & 0.791 & $83(19.2 \%)$ \\
\hline Stroke & $841 / 841$ & $7(2.2 \%)$ & $17(3.7 \%)$ & $1(1.7 \%)$ & 0.395 & $23(5.3 \%)$ \\
\hline Peripheral artery disease & $841 / 841$ & $3(0.9 \%)$ & $6(1.3 \%)$ & $2(3.3 \%)$ & 0.325 & $13(3.0 \%)$ \\
\hline Chronic kidney disease & $841 / 841$ & $2(0.6 \%)$ & $4(0.9 \%)$ & $0(0 \%)$ & 0.733 & $14(3.2 \%)$ \\
\hline Chronic obstructive pulmonary disease & $841 / 841$ & $2(0.6 \%)$ & $6(1.3 \%)$ & $1(1.7 \%)$ & 0.596 & $8(1.8 \%)$ \\
\hline \multicolumn{7}{|l|}{ Medications on discharge } \\
\hline Aspirin & $841 / 841$ & $271(84.7 \%)$ & $391(84.8 \%)$ & $47(78.3 \%)$ & 0.418 & $280(64.7 \%)$ \\
\hline Clopidogrel & $841 / 841$ & $175(54.7 \%)$ & $282(61.2 \%)$ & $35(58.3 \%)$ & 0.195 & $190(43.9 \%)$ \\
\hline Ticagrelor & $841 / 841$ & $92(28.8 \%)$ & $82(17.8 \%)$ & $11(18.3 \%)$ & 0.001 & $56(12.9 \%)$ \\
\hline Prasugrel & $841 / 841$ & $2(0.6 \%)$ & $5(1.1 \%)$ & $0(0 \%)$ & 0.599 & $8(1.9 \%)$ \\
\hline ACE-inhibitors & $841 / 841$ & $218(68.1 \%)$ & $278(60.3 \%)$ & $39(65.0 \%)$ & 0.08 & $202(46.7 \%)$ \\
\hline ARB & $841 / 841$ & $44(13.8 \%)$ & $96(20.8 \%)$ & $4(6.7 \%)$ & 0.003 & 95 (21.9\%) \\
\hline Beta-receptor blockers & $841 / 841$ & 278 (86.9\%) & $394(85.5 \%)$ & $46(76.7 \%)$ & 0.121 & $277(64.0 \%)$ \\
\hline Statins & $841 / 841$ & $276(86.3 \%)$ & $393(85.3 \%)$ & $47(78.3 \%)$ & 0.285 & $271(62.6 \%)$ \\
\hline Dihydropyridines & $841 / 841$ & $60(18.8 \%)$ & $88(19.1 \%)$ & $13(21.7 \%)$ & 0.870 & $111(25.6 \%)$ \\
\hline Diuretics & $841 / 841$ & $49(15.3 \%)$ & $80(17.4 \%)$ & $10(16.7 \%)$ & 0.752 & $101(23.3 \%)$ \\
\hline Warfarin & $841 / 841$ & $11(3.4 \%)$ & $26(5.6 \%)$ & $5(8.3 \%)$ & 0.178 & $29(6.7 \%)$ \\
\hline
\end{tabular}

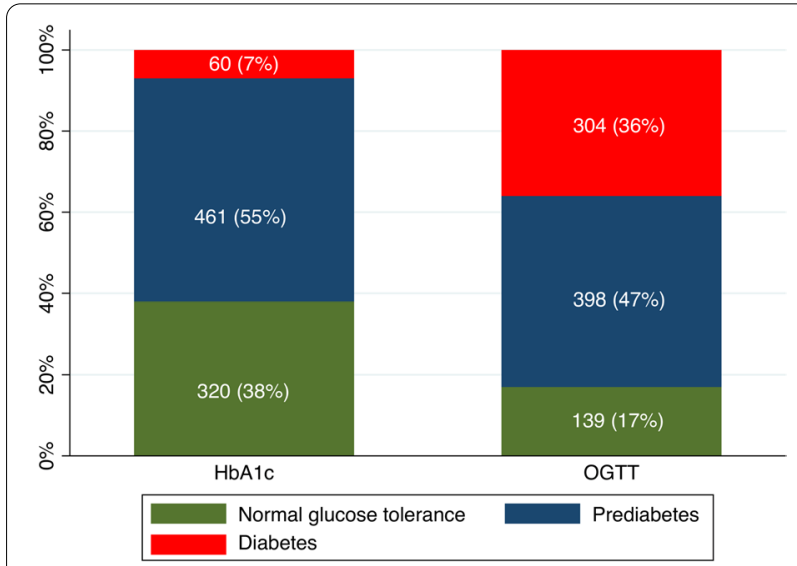

Fig. 1 Number and percentage of patients in different glucose groups according to OGTT and $\mathrm{HbA} 1 \mathrm{c}$ results (ADA criteria)
$\mathrm{N}=754$

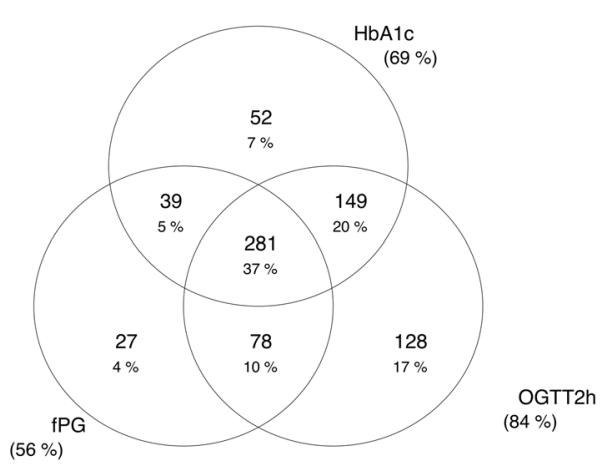

Fig. 2 Venn diagram showing proportions and their overlap between $\mathrm{fPG}, 2 \mathrm{~h}-\mathrm{PG}$ and $\mathrm{HbA} 1 \mathrm{c}$ in classifying dysglycaemia (prediabetes or diabetes) in the 754 out of 841 patients who had dysglycaemia according to either OGTT or $\mathrm{HbA} 1 \mathrm{c}$ result (ADA criteria) 
Table 3 Absolute numbers (\%), event rates per 100 person-years and hazard ratios for the combined event (CE; first of myocardial infarction, hospitalisation for heart failure, ischaemic stroke or mortality) in 841 patients stratified by glucose perturbation group according to OGTT and $\mathrm{HbA} 1 \mathrm{c}$ results (ADA criteria)

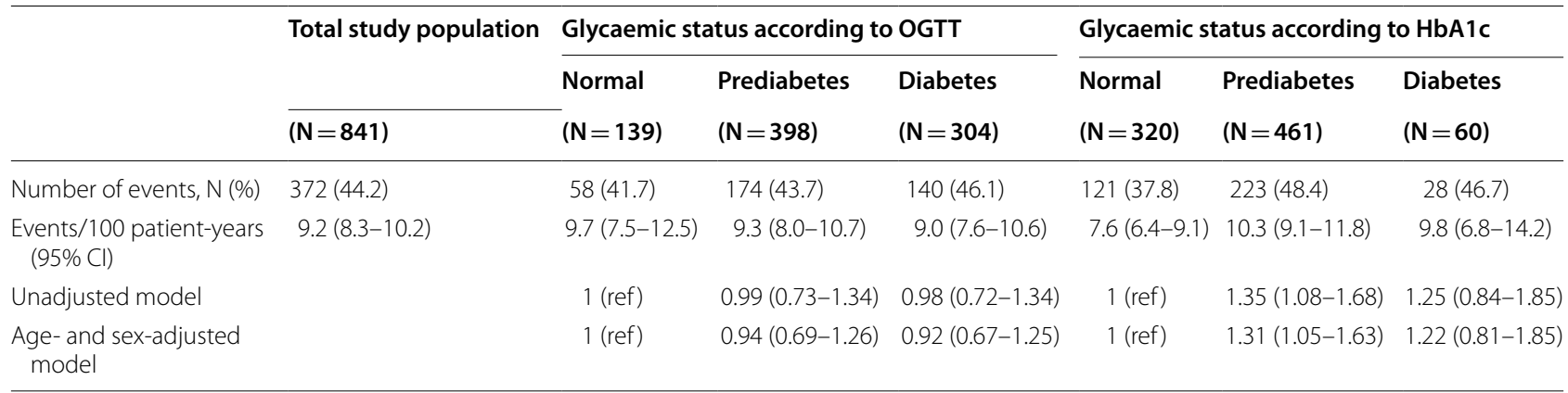

$\mathrm{Cl}$ : confidence interval; ref: reference category; N: number of patients

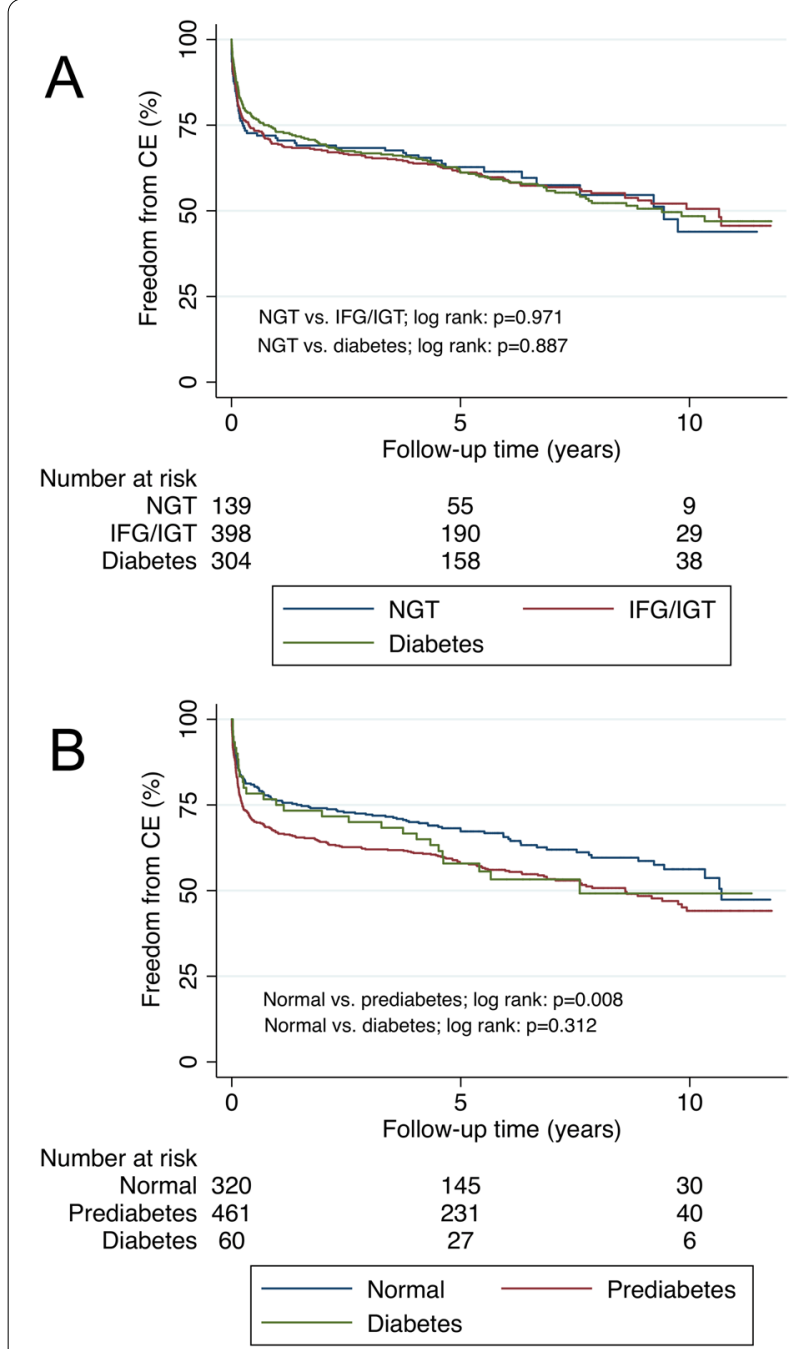

Fig. 3 Kaplan-Meier curve showing time to freedom from combined event (CE; first of myocardial infarction, hospitalisation for heart failure, ischaemic stroke or mortality) for different categories of dysglycaemia according to $\mathbf{A}$ Fasting and 2 h-PG results (OGTT) and B HbA1c
Similarly, there were no significant differences in the incidence rates for the CE or HRs between the three patient groups when looking at the total number of patients who underwent an OGTT $(\mathrm{n}=1684)$ (Additional file 1: Table S5).

\section{Event rates and long-term outcome in patient groups according to $\mathrm{HbA} 1 \mathrm{c}$ results}

When patients were stratified by glucose groups according to $\mathrm{HbA1c}$ results, the $\mathrm{CE}$ of first of myocardial infarction, ischaemic stroke, hospitalisation for heart failure or mortality occurred in 121 (37.8\%) patients with normoglycaemia (HbA1c $<39 \mathrm{mmol} / \mathrm{mol})$, in $223(48.4 \%)$ patients with prediabetes (HbAlc $39-47 \mathrm{mmol} / \mathrm{mol}$ ) and in $28(46.7 \%)$ patients with newly discovered diabetes $(\mathrm{HbA} 1 \mathrm{c} \geq 48 \mathrm{mmol} / \mathrm{mol})$. Patients with prediabetes had a poorer prognosis, with significantly higher risk for cardiovascular events and mortality. When adjusted for age and sex, the HR for the combined event was 1.31 (CI 1.05-1.63) compared to the group with normal HbA1c (Table 3). Figure 3B illustrates a Kaplan-Meier curve with freedom from the $C E$ between the three patient groups and Additional file 1: Figure S2B illustrates a Kaplan Meier curve with freedom from all-cause mortality, categorised according to HbA1c results.

\section{Comparison of associated risk for combined event by different glucose and $\mathrm{HbA} 1 \mathrm{c}$ thresholds according to ADA and WHO criteria}

Figure 4 depicts a forest plot with HRs for the CE, adjusted for age and sex, in patients categorised into different glucose groups according to both ADA and WHO criteria. Results are also shown with patients stratified into three or two groups according to fPG, 2 h-PG and $\mathrm{HbA1c}$ results. Similar findings are demonstrated, regardless of whether ADA or WHO criteria were used, when patients were divided into three groups, with 


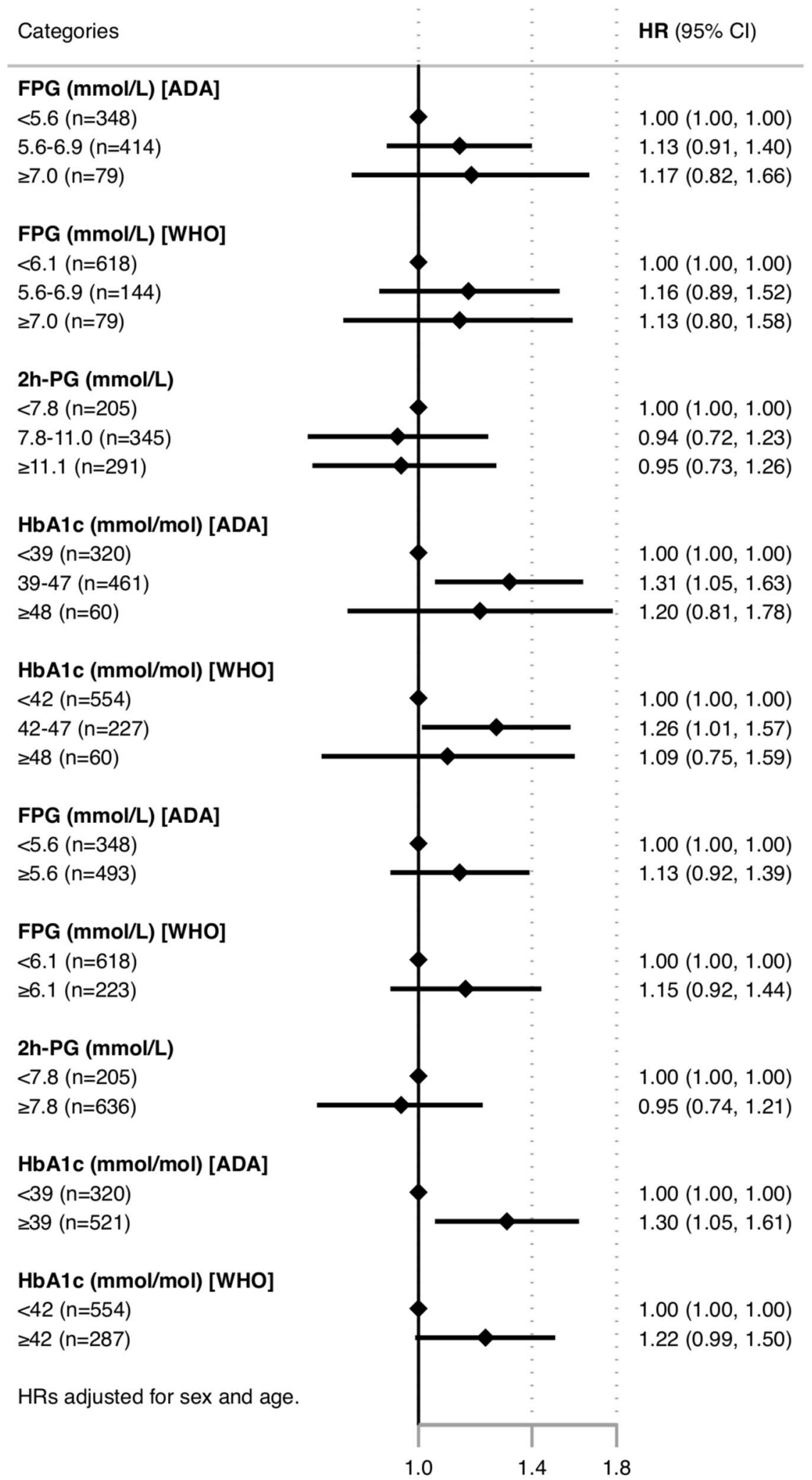

Fig. 4 Forest plot with HR (95\% Cl) for the prognostic value of FPG, 2 h-PG and HbA1c for the combined event (first of mortality, heart failure, ischaemic stroke, and myocardial infarction) using different cut-offs for dysglycaemia according to ADA and WHO definitions 
worse outcomes in the prediabetes group categorised by HbA1c. When patients were divided into two groups (normal or abnormal) there was a statistically significant increased risk for $\mathrm{CE}$ only when using the HbA1c cutoff of $39 \mathrm{mmol} / \mathrm{mol}$, i.e., ADA criteria. A similar trend was seen when using the HbA1c cut-off of $42 \mathrm{mmol} / \mathrm{mol}$ (WHO), but this did not reach statistical significance.

\section{Discussion}

The present study confirms that approximately two thirds of patients admitted with an AMI have previously undetected glucose disturbances. An OGTT classified 83\% of our study population as having dysglycaemia, while a single $\mathrm{HbA} 1 \mathrm{c}$ classified $62 \%$. It is well known that a large number of patients with type 2 diabetes remain undiagnosed for many years and that a long duration of undetected hyperglycaemia may have detrimental effects on both micro- and macrovascular complications [20]. By screening for glucose disturbances in patients with AMI, lifestyle modification and pharmacological treatment can be initiated earlier. In a recently published register study, it was shown that patients with prediabetes treated with metformin have an improved outcome [21]. However, whether early lifestyle and medical interventions have a positive effect on short- and/or long-term outcome needs to be further established in randomised intervention studies.

The latest European Society of Cardiology guidelines on diabetes, pre-diabetes and cardiovascular diseases recommend that screening for diabetes in patients with cardiovascular disease should begin with fPG and HbA1c and, only if needed, complemented with an OGTT [15]. This recommendation seems to be supported by the results of our study, as an OGTT (fPG + 2 h-PG) identifies only $10 \%$ more patients as having dysglycaemia compared to the combination of fPG and HbA1c. Moreover, an OGTT should be performed no earlier than four days after an AMI to increase its reliability [15]. This is becoming more difficult due to the decreasing length of hospital stay for AMI-Sweden, for example, had an average length of stay of 4.1 days in 2017 [22]. Previous studies have shown that an OGTT is more sensitive in identifying dysglycaemia in populations with coronary artery disease [12, 23] and acute stroke [24]. However, in a population with overweight or obesity, HbA1c identified more patients with prediabetes than an OGTT [25].

As has been noted in other studies, [12, 25] the OGTT and HbA1c seem to identify different highat-risk populations with a relatively low concordance between them. As such, it is particularly clinically relevant to identify the test which has the most predictive value for important patient outcomes in patients with AMI. Results from previous studies have been conflicting $[13,26,27]$. In our study, HbA1c was a better predictor of future cardiovascular events than either fPG or $2 \mathrm{~h}-\mathrm{PG}$. The OGTT did not predict mortality or combined events in any of the glucose groups-neither in the group of patients who had HbA1c simultaneously analysed $(n=841)$, nor in the larger group of patients who only underwent testing with an OGTT $(\mathrm{n}=1684)$. This finding is in agreement with previous studies that showed that HbA1c was better than fPG in predicting the risk for all-cause mortality and cardiovascular events [26]. However, a report from EUROASPIRE IV, concluded that only 2 h-PG levels and not fPG or HbA1c in patients with stable coronary artery disease predicted future cardiovascular events [13]. Similarly, another study in patients with AMI showed that $2 \mathrm{~h}-\mathrm{PG}$, but not PPG was predictive for the risk for allcause mortality or reinfarction. In this study HbA1c was not studied [27].

Interestingly, when patients were categorised according to HbA1c levels, the group of patients with prediabetes had a higher incidence of death, myocardial infarction, ischaemic stroke and hospitalisation for heart failure than patients who were categorised as having diabetes. Patients with prediabetes (HbA1c 39 to $47 \mathrm{mmol} / \mathrm{mol}$ ) had a $30 \%$ higher risk for the combined outcome compared to patients with normoglycemia ( $\mathrm{HbA} 1 \mathrm{c}<39 \mathrm{mmol} / \mathrm{L}$ ). Although point estimates suggested a higher risk for the combined event also in patients with newly detected diabetes $(\mathrm{HbA} 1 \mathrm{c} \geq 48 \mathrm{mmol} / \mathrm{mol})$ compared to patients with normoglycaemia, the confidence intervals were wide and the result non-significant.

There might be different explanations for these results. In this observational single-centre study, performed in patients treated with routine clinical care for AMI, there was a high attention and interest in disturbed glucose metabolism and its effects on cardiovascular outcomes. Therefore, screening with an OGTT in patients with AMI as a standard was introduced in 2006. Patients with glucose disturbances, in particular patients with newly detected glucose abnormalities by an OGTT, were offered lifestyle modification counselling and were frequently referred to their general practitioner for a follow-up diagnostic OGTT and ongoing management. This may have resulted in an improved secondary prevention, which may have been superior in patients with diabetes compared to the patients with prediabetes, i.e., patients with diagnosed diabetes were more likely to be treated with guideline-recommended cardiovascular drugs which would lower their risk for adverse outcomes. This intervention was however not performed in a randomised fashion, and we have no information on lifestyle modification achievement or long-term medical treatment. 
Another explanation could be that, even though $\mathrm{HbA1c}$ was recommended as a diagnostic tool by ADA in $2009^{9}$ and by WHO in 2011 [28], it was not used routinely in the screening of patients with cardiovascular disease in Sweden until 2014. During the inclusion period for this study, 2006 to 2013, cardiologists were not familiar with considering $\mathrm{HbA1c}$ results as a diagnostic tool for type 2 diabetes mellitus, meaning that a pathological OGTT result was more likely to attract attention from the attending physician than a HbA1c in the prediabetic range.

\section{Strengths and limitations}

This study was conducted at one centre and is an observational study and should be reproduced in other hospitals for evaluation of generalisability. Outcomes were retrieved from Swedish national registers, which have been shown to have high validity [29]. There was virtually no loss to follow-up as every individual in Sweden has their own unique personal identity number. We used a standardised method to measure HbA1c and all OGTTs were performed according to WHO guidelines. All samples were analysed in one single accredited laboratory ensuring a high validity of the measures obtained in this study.

We only had information on laboratory values and medical treatment at the time of the index AMI. The effects of lifestyle modification and secondary preventive medical treatment that could affect the outcome could not be analysed. At the beginning of the inclusion period, when HbA1c was not used routinely as a diagnostic tool, most likely a selection bias was introduced, as there was a tendency to have an additional HbA1c analysed in those with a pathologic OGTT. Furthermore, with missing data for a number of variables, we only adjusted data for age and sex, therefore residual confounding may be present.

\section{Conclusions}

In conclusion, we confirm that two thirds of patients with AMI with no previously known history of diabetes have dysglycaemia when investigated with either an OGTT or HbA1c, illustrating the importance of screening for diabetes in this population. In this observational study, only dysglycaemia classified by a HbA1c, but not an OGTT, analysed at the time of the AMI, added predictive value to the long-term prognosis. Our findings seem to support the latest guidelines recommending to primarily use fPG and HbA1c in screening for diabetes after AMI and, only if needed, complement with an OGTT. However, increased awareness of glucose disturbances revealed by an OGTT and a special cardiac rehabilitation program for this group, might have influenced the results.

\section{Abbreviations}

OGTT: Oral glucose tolerance test; HbA1c: Haemoglobin A1c; AMI: Acute myocardial infarction; NGT: Normal glucose tolerance; HR: Hazard ratio; Cl: Confidence interval; ADA: American Diabetes Association; WHO: World Health Organisation; IFG: Impaired fasting glycaemia; IGT: Impaired glucose tolerance; fPG: Fasting glucose; 2 h-PG: 2-Hour postload glucose; CE: Combined event.

\section{Supplementary Information}

The online version contains supplementary material available at https://doi. org/10.1186/s12933-021-01315-5.

Additional file 1: Figure S1. Flowchart for the study population. Table S1. Definitions of normal glycaemic status and dysglycaemia according to ADA and WHO criteria. Table S2. List of ICD-10 codes and definitions used in our study. Table $\mathbf{S 3}$. Baseline characteristics of all patients screened with OGTT $(n=1684)$ stratified by glycaemic status according to ADA criteria. Table S4. Absolute numbers (\%), event rates per 100 person-years and hazard ratios for all-cause mortality in 841 patients stratified by glucose perturbation group according to OGTT and $\mathrm{HbA} 1 \mathrm{c}$ results (ADA criteria). Figure S2. Kaplan-Meier curve showing time to freedom from all-cause mortality for different categories of dysglycaemia according to A. Fasting and 2-hour post-load glucose results (OGTT) and B. HbA1c. Table S5. Absolute numbers (\%), event rates per 100 person-years and hazard ratios for the combined event (CE; first of myocardial infarction, hospitalisation for heart failure, ischaemic stroke or mortality) in 1684 patients stratified by glucose perturbation group according to OGTT (ADA criteria).

Acknowledgements

Not applicable.

\section{Authors' contributions}

All authors participated in the research design, to the interpretation of data, critical revision of the manuscript, provided important intellectual content and gave the final approval. SK and CD contributed equally. SK and CD wrote the first draft of the manuscript. SK performed statistical analyses after discussion with all authors. All authors agree to be accountable for all aspects of work ensuring integrity and accuracy. All authors read and approved the final manuscript.

\section{Funding}

Open access funding provided by Karolinska Institute. This study was supported by funding from Stockholm County Council (ALF).

\section{Availability of data and materials}

The datasets used and/or analysed during the current study are available from the corresponding author on reasonable request.

\section{Declarations}

Ethics approval and consent to participate

This study was approved by the regional research ethics committee in Stockholm, Sweden (reg. no 2014/338-31/2 and 2018/1321-32).

\section{Consent for publication}

Not applicable.

\section{Competing interests}

The authors declare that they have no conflicts of interest concerning this study. Dr. Karayiannides reports personal fees from Astra Zeneca, Boehringer Ingelheim and Novo Nordisk, outside the submitted work. Dr. Djupsjö, Dr. Kuhl and Dr. Hofman-Bang have nothing to disclose. Prof. Norhammar reports 
personal fees from Astra Zeneca, MSD, Boehringer Ingelheim, Lilly and Novo Nordisk, outside the submitted work. Prof. Holzmann reports personal fees from Idorsia, outside the submitted work. Dr. Lundman reports personal fees from Boehringer Ingelheim, Sanofi and Novo Nordisk, outside the submitted work.

\section{Author details}

'Department of Clinical Sciences, Karolinska Institutet, Danderyd Hospital, SE-182 88 Stockholm, Sweden. ${ }^{2}$ Centre for Diabetes, Academic Specialist Centre, Region Stockholm, Sweden. ${ }^{3}$ Department of Medicine (K2), Karolinska Institutet, Stockholm, Sweden. ${ }^{4}$ Heart, Vascular and Neuro Theme, Karolinska University Hospital, Stockholm, Sweden. ${ }^{5}$ Division of Medicine, Danderyd University Hospital, Stockholm, Sweden. ${ }^{6}$ Department of Cardiology, Danderyd University Hospital, Stockholm, Sweden. ${ }^{7}$ Capio S:t Görans Hospital, Stockholm, Sweden. ${ }^{8}$ Theme of Emergency and Reparative Medicine, Karolinska University Hospital, Stockholm, Sweden.

Received: 28 March 2021 Accepted: 5 June 2021 Published online: 14 June 2021

\section{References}

1. Grundy SM, Pasternak R, Greenland P, et al. Assessment of cardiovascular risk by use of multiple-risk-factor assessment equations. J Am Coll Cardiol. 1999;34:1348-59.

2. Brunner EJ, Shipley MJ, Witte DR, et al. Relation between blood glucose and coronary mortality over 33 years in the Whitehall Study. Diabetes Care. 2005:29:26-31.

3. Norhammar A, Mellbin L, Cosentino F. Diabetes: prevalence, prognosis and management of a potent cardiovascular risk factor. Eur J Prev Cardiol. 2017;24:52-60.

4. Ritsinger $\mathrm{V}$, Tanoglidi $\mathrm{E}$, Malmberg $\mathrm{K}$, et al. Sustained prognostic implications of newly detected glucose abnormalities in patients with acute myocardial infarction: long-term follow-up of the Glucose Tolerance in Patients with Acute Myocardial Infarction cohort. Diabetes Vasc Dis Re. 2014;12:23-32

5. Otten R, Kline-Rogers E, Meier DJ, et al. Impact of pre-diabetic state on clinical outcomes in patients with acute coronary syndrome. Heart. 2005;91:1466.

6. Bartnik M, Malmberg K, Norhammar A, et al. Newly detected abnormal glucose tolerance: an important predictor of long-term outcome after myocardial infarction. Eur Heart J. 2004;25:1990-7.

7. Collaboration ERF, Sarwar N, Gao P, et al. Diabetes mellitus, fasting blood glucose concentration, and risk of vascular disease: a collaborative metaanalysis of 102 prospective studies. Lancet. 2010:375:2215-22.

8. Alberti KGMM, Zimmet PZ, Consultation W. Definition, diagnosis and classification of diabetes mellitus and its complications. Part 1: diagnosis and classification of diabetes mellitus. Provisional report of a WHO Consultation. Diabetic Med. 1998;15:539-53.

9. Committee TIE. International Expert Committee Report on the Role of the A1C Assay in the Diagnosis of Diabetes. Diabetes Care. 2009;32:1327-34.

10. Zimmet $P$, Alberti KG, Magliano DJ, et al. Diabetes mellitus statistics on prevalence and mortality: facts and fallacies. Nat Rev Endocrinol. 2016;12:616-22

11. Danaei G, Fahimi S, Lu Y, et al. Effects of diabetes definition on global surveillance of diabetes prevalence and diagnosis: a pooled analysis of 96 population-based studies with 331288 participants. Lancet Diabetes Endocrinol. 2015;3:624-37.

12. Gyberg V, Bacquer DD, Kotseva K, et al. Screening for dysglycaemia in patients with coronary artery disease as reflected by fasting glucose, oral glucose tolerance test, and $\mathrm{HbA} 1 \mathrm{c}$ : a report from EUROASPIRE IV-a survey from the European Society of Cardiology. Eur Heart J. 2015;36:1171-7.
13. Shahim B, Bacquer DD, Backer GD, et al. The prognostic value of fasting plasma glucose, two-hour postload glucose, and $\mathrm{HbA} 1 \mathrm{c}$ in patients with coronary artery disease: a report from EUROASPIRE IV. Diabetes Care. 2017;40:dc170245.

14. Nakagami T, Group DS. Hyperglycaemia and mortality from all causes and from cardiovascular disease in five populations of Asian origin. Diabetologia. 2004:47:385-94.

15. Cosentino F, Grant PJ, Aboyans V, et al. 2019 ESC Guidelines on diabetes, pre-diabetes, and cardiovascular diseases developed in collaboration with the EASDThe Task Force for diabetes, pre-diabetes, and cardiovascular diseases of the European Society of Cardiology (ESC) and the European Association for the Study of Diabetes (EASD). Eur Heart J. 2019:41:255-323.

16. Norhammar A, Tenerz Å, Nilsson G, et al. Glucose metabolism in patients with acute myocardial infarction and no previous diagnosis of diabetes mellitus: a prospective study. Lancet. 2002;359:2140-4.

17. Jernberg T, Attebring MF, Hambraeus K, et al. The Swedish web-system for enhancement and development of evidence-based care in heart disease evaluated according to recommended therapies (SWEDEHEART). Heart. 2010;96:1617.

18. Ludvigsson JF, Otterblad-Olausson P, Pettersson BU, et al. The Swedish personal identity number: possibilities and pitfalls in healthcare and medical research. Eur J Epidemiol. 2009;24:659-67.

19. Association AD. 2. Classification and diagnosis of diabetes: standards of medical care in diabetes-2021. Diabetes Care. 2020:44:S15-33.

20. Canto ED, Ceriello A, Rydén L, et al. Diabetes as a cardiovascular risk factor: an overview of global trends of macro and micro vascular complications. Eur J Prev Cardiol. 2019;26:25-32.

21. Ritsinger $V$, Lagerqvist $B$, Lundman $P$, et al. Diabetes, metformin and glucose lowering therapies after myocardial infarction: Insights from the SWEDEHEART registry. Diabetes Vasc Dis Re. 2020;17:1479164120973676.

22. OECD. Health at a Glance 2019, https://www.oecd-ilibrary.org/content/ publication/4dd50c09-en (2019).

23. Bartnik M, Rydén L, Malmberg K, et al. Oral glucose tolerance test is needed for appropriate classification of glucose regulation in patients with coronary artery disease: a report from the Euro Heart Survey on Diabetes and the Heart. Heart. 2007;93:72.

24. Matz K, Tuomilehto J, Teuschl Y, et al. Comparison of oral glucose tolerance test and $\mathrm{HbA} 1 \mathrm{c}$ in detection of disorders of glucose metabolism in patients with acute stroke. Cardiovasc Diabetol. 2020;19:204.

25. Chatzianagnostou K, Vigna L, Piazza SD, et al. Low concordance between $\mathrm{HbA} 1 \mathrm{c}$ and OGTT to diagnose prediabetes and diabetes in overweight or obesity. Clin Endocrinol. 2019;91:411-6.

26. Selvin E, Steffes MW, Zhu H, et al. Glycated hemoglobin, diabetes, and cardiovascular risk in nondiabetic adults. New Engl J Med. 2010:362:800-11.

27. Chattopadhyay S, George A, John J, et al. Adjustment of the GRACE score by 2-hour post-load glucose improves prediction of long-term major adverse cardiac events in acute coronary syndrome in patients without known diabetes. Eur Heart J. 2018;39:2740-5.

28. Use of glycated haemoglobin ( $\mathrm{HbA} 1 \mathrm{c})$ in the diagnosis of diabetes mellitus. Diabetes Res Clin Pr 2011; 93: 299-309.

29. Ludvigsson JF, Andersson E, Ekbom A, et al. External review and validation of the Swedish national inpatient register. BMC Public Health. 2011;11:450.

\section{Publisher's Note}

Springer Nature remains neutral with regard to jurisdictional claims in published maps and institutional affiliations. 